Purdue University Purdue e-Pubs

$12-1997$

\title{
Plane Wave Reflection Coefficient Estimation by Use of Spatial Parametric Signal Modeling
}

J. Stuart Bolton

Purdue University, bolton@purdue.edu

Hyu-Sang Kwon

Follow this and additional works at: http://docs.lib.purdue.edu/herrick

Bolton, J. Stuart and Kwon, Hyu-Sang, "Plane Wave Reflection Coefficient Estimation by Use of Spatial Parametric Signal Modeling" (1997). Publications of the Ray W. Herrick Laboratories. Paper 21.

http://docs.lib.purdue.edu/herrick/21

This document has been made available through Purdue e-Pubs, a service of the Purdue University Libraries. Please contact epubs@purdue.edu for additional information. 


\section{Plane Wave Reflection Coefficient Estimation By Use Of Spatial Parametric Signal Modeling}

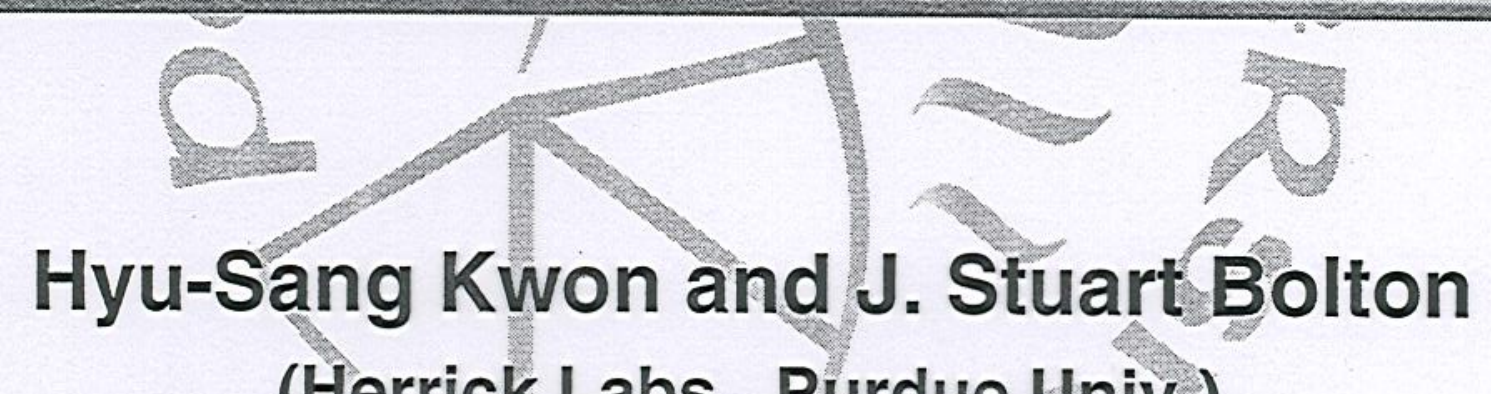

(Herrick Labs., Purdue Univ:) 


\section{INTRODUCTION}

- How can we measure the reflection coefficient? plane wave

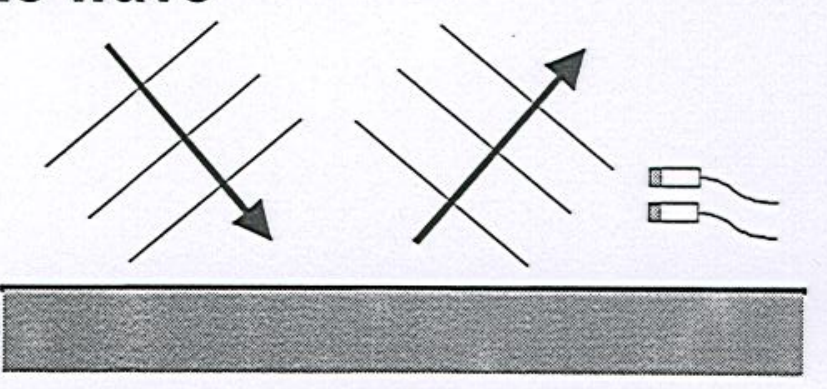

incoming and outgoing wave decomposition by two microphones axisymetric source

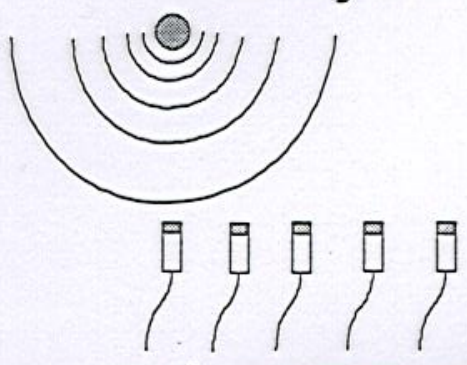

plane wave decomposition by linear microphone array
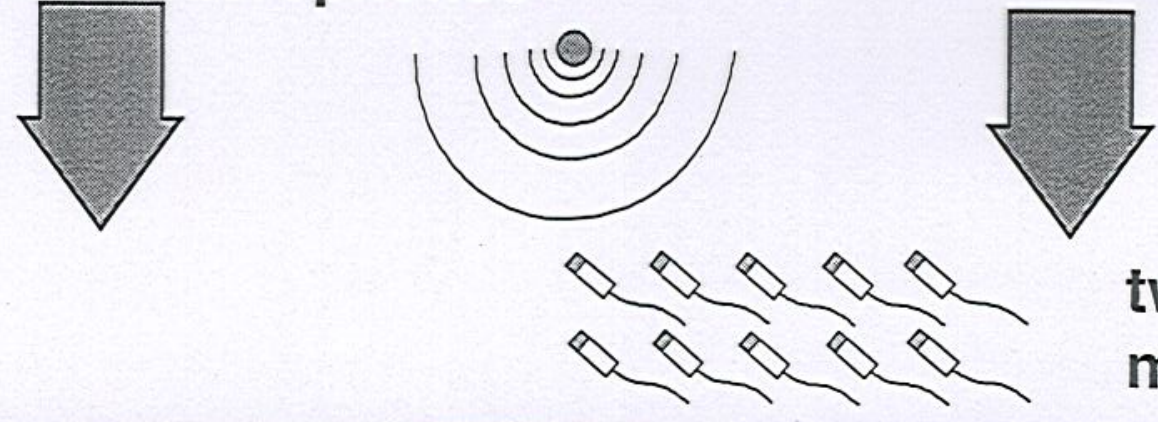

two plane linear microphone array

reflection coefficient measurement w.r.t incident angle by wave decomposition theory 


\section{WAVE NUMBER SPECTRUM}

- Plane wave decomposition theory

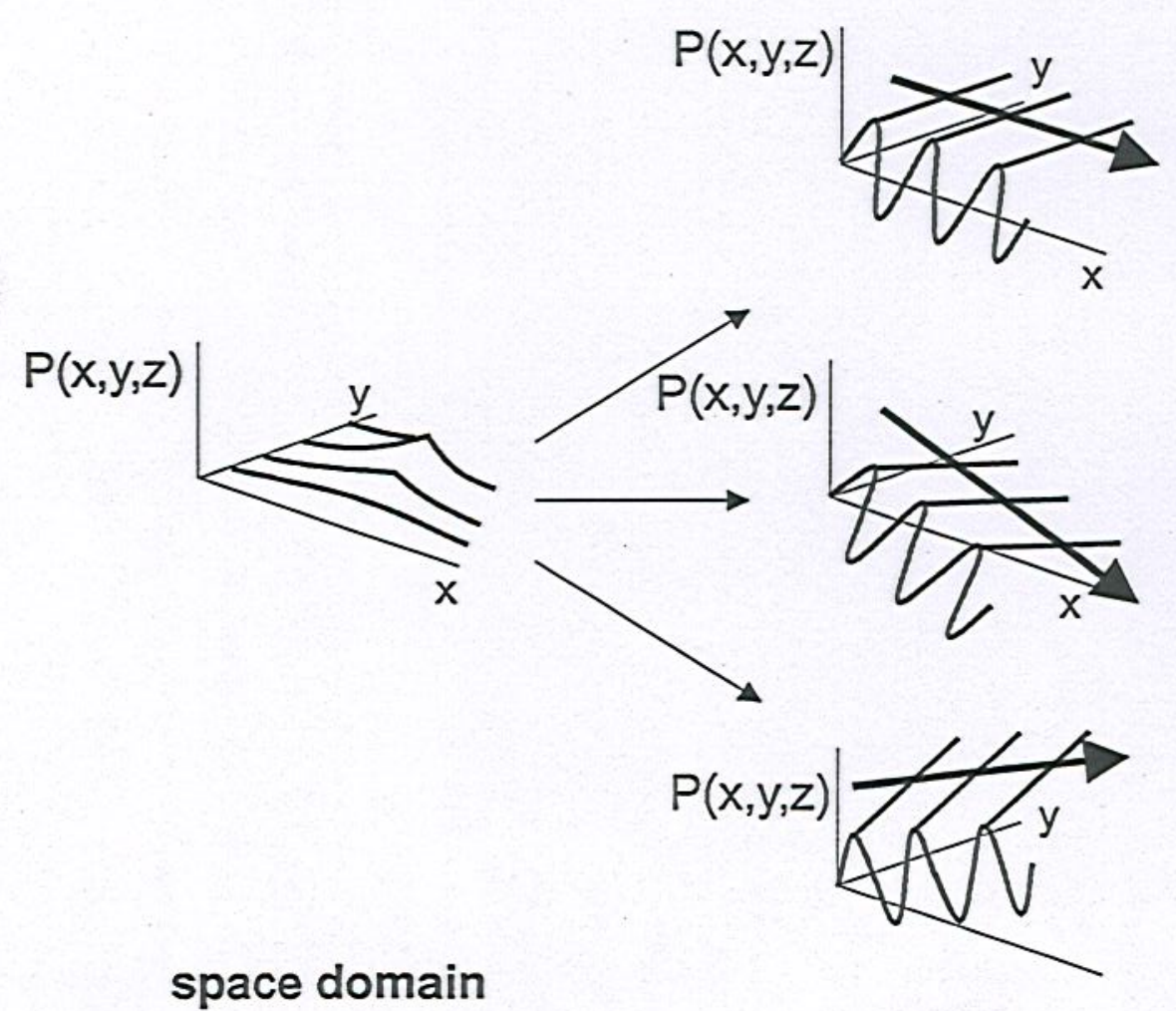

Spatial Fourier transform
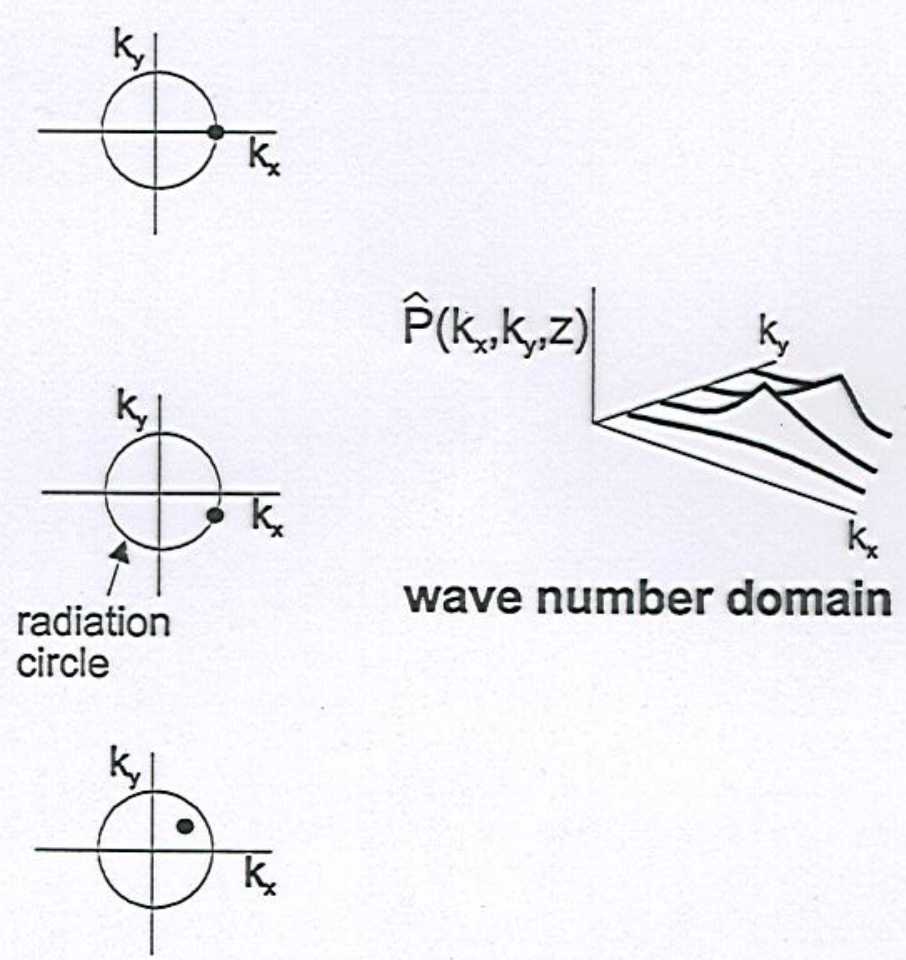

wave number domain 


\section{ZEROTH ORDER HANKEL TRANSFORM}

- An alternative of Fourier transform

- Axisymmetric field

- Corresponds to 2 dim. spatial Fourier transform

Non-axisymmetric field

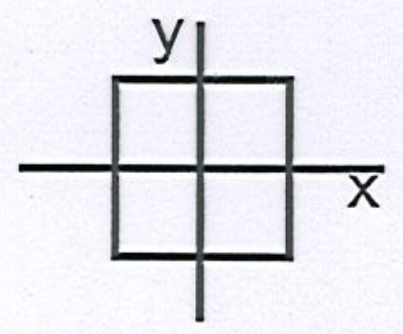<smiles></smiles>

Non-axisymmetric wave number spectrum

2 dimensional spatial Fourier transform

Axisymmetric

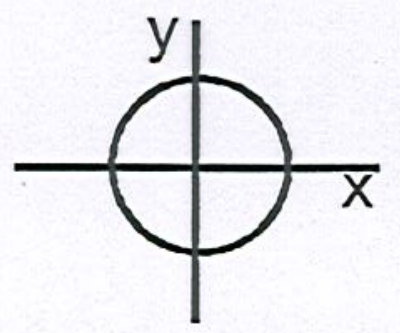

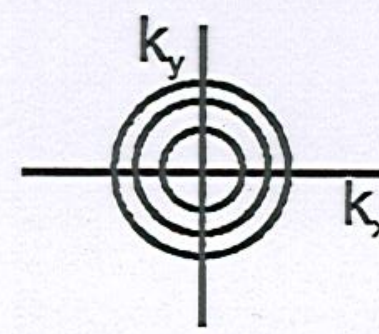

Axisymmetric wave number spectrum field

$$
\begin{array}{ll}
\text { space domain } & \begin{array}{l}
\text { wave number } \\
\text { domain }
\end{array} \\
r \text { dependent } & k_{r} \text { dependent }
\end{array}
$$




\section{REFLECTION COEFFICIENT}

- Procedure

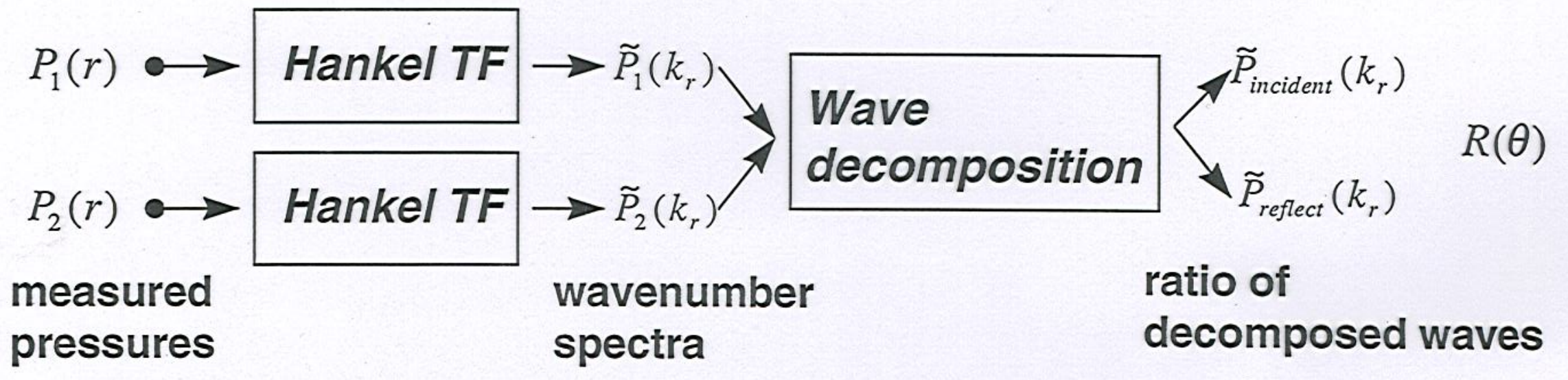

- Zeroth order Hankel transform

- Definition: $\tilde{X}\left(k_{r}\right)=\int_{0}^{\infty} r X(r) J_{0}\left(k_{r} r\right) d r$

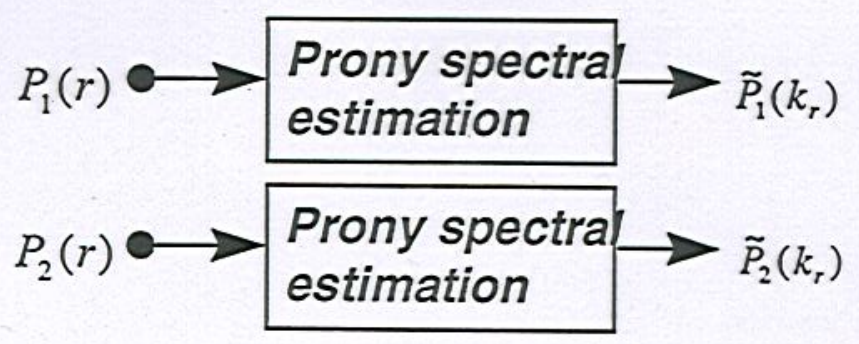

- Wave number and angle relation

$$
k_{r}=\sqrt{k_{x}^{2}+k_{y}^{2}} \quad \theta=\cos ^{-1}\left(\frac{\sqrt{k^{2}-k_{r}^{2}}}{k}\right)
$$




\section{PRONY APPROACH}

- Finite measurement aperture

- Finite size of anechoic room \& material

- Finite number of measuring microphones

- DHT(Discrete Hankel Transform)

- Leakage \& poor spectral resolution

- Less information

$$
\frac{\Delta k_{r}}{k}=\frac{\lambda}{N \Delta r}
$$

- Parametric spectral estimation

- Prony model: damped harmonic signal

- High resolution of reflection coefficient

$$
P(r)=\sum_{n=1}^{N} A_{n} e^{-\alpha_{n} r}
$$

- Less measurement positions

- Model order selection: by using input white noise variance

$$
\begin{aligned}
& X(z)=H(z) U(z), \quad \rho_{w}=P_{U U}(z) \\
& \text { constant }
\end{aligned}
$$

Ref.: Akaike Information Criterion, etc. 


\section{SIMULATION}

- Simulation setup

Monopole or dipole

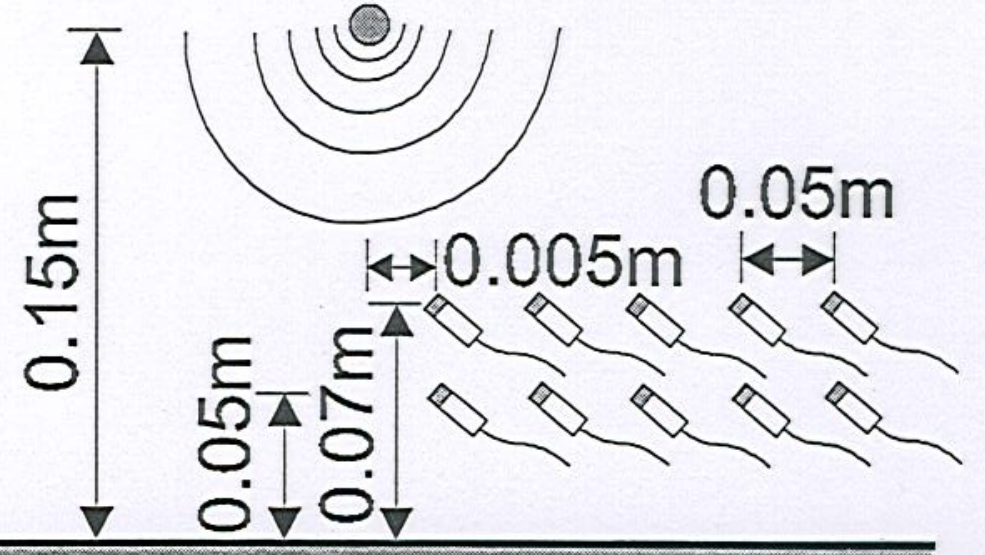

Frequency $=343 \mathrm{~Hz}$

Wavelength $=1 \mathrm{~m}$

No. of data $=50$ per line

- Monopole, dipole simulation

Reflection coefficients calculation

Rigid wall, $\mathrm{R}=1$ or

No wall, $R=0$

- Discrete Hankel Transform

- Prony spectral estimation

- No wall, rigid wall simulation 


\section{MONOPOLE CASE}
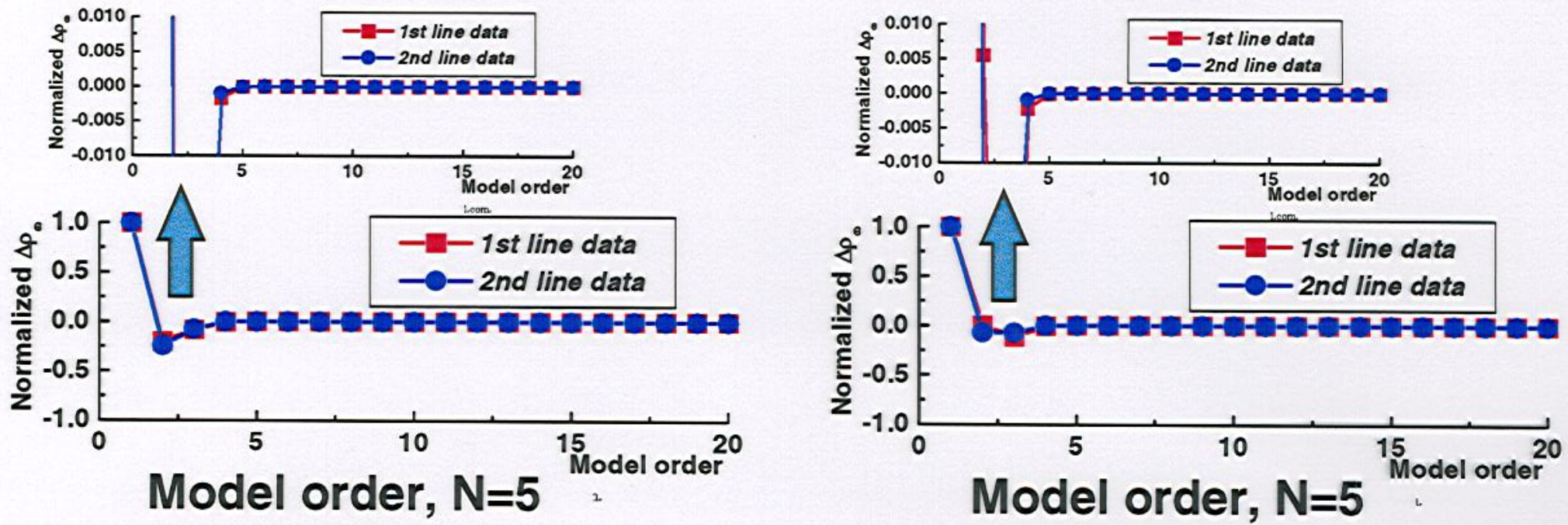

No. of poles $=6$

No. of poles $=6$
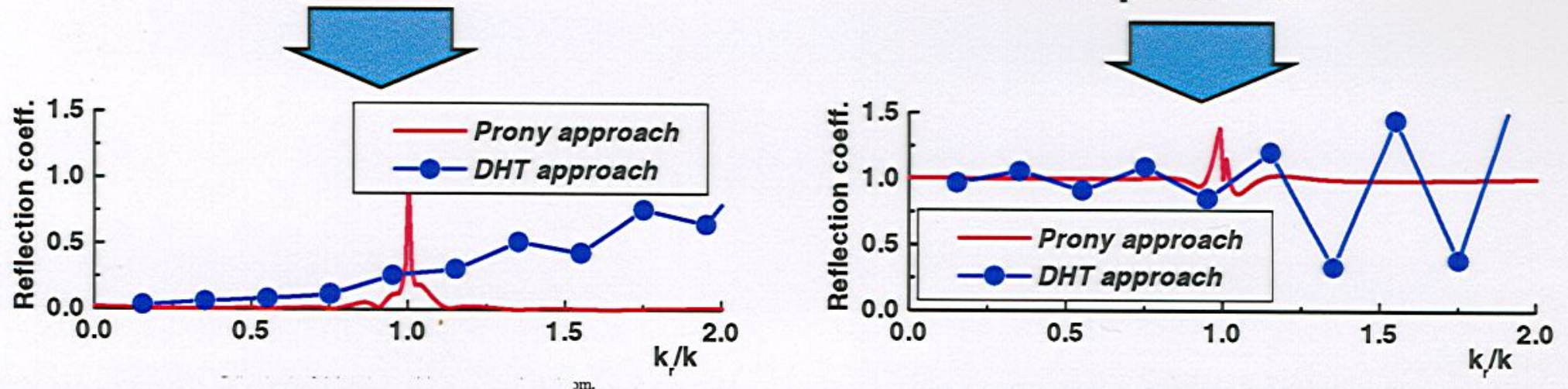

Monopole without reflecting wall

Monopole with rigid reflecting wall

\section{Purdue University}




\section{DIPOLE CASE}

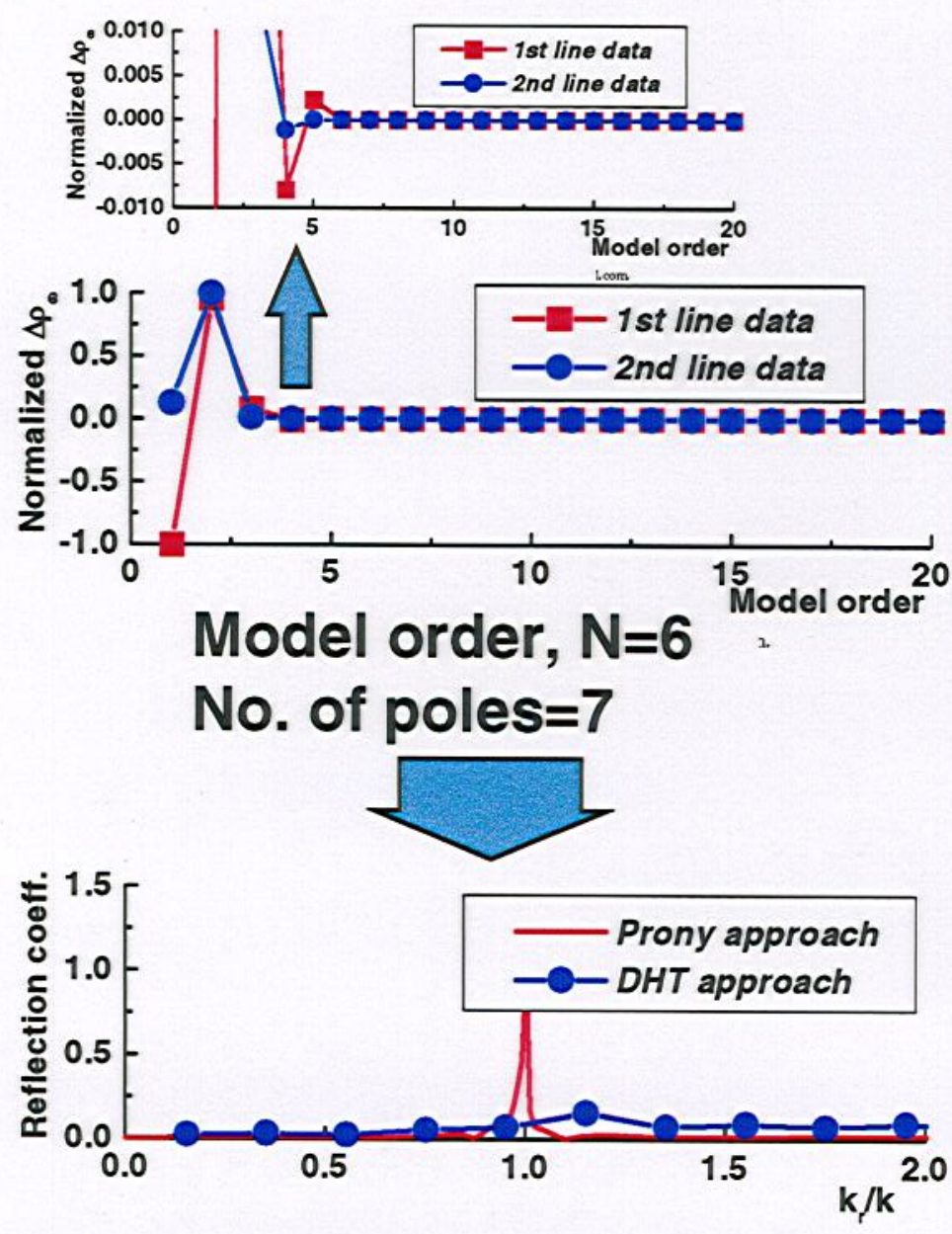

Dipole without reflecting wall

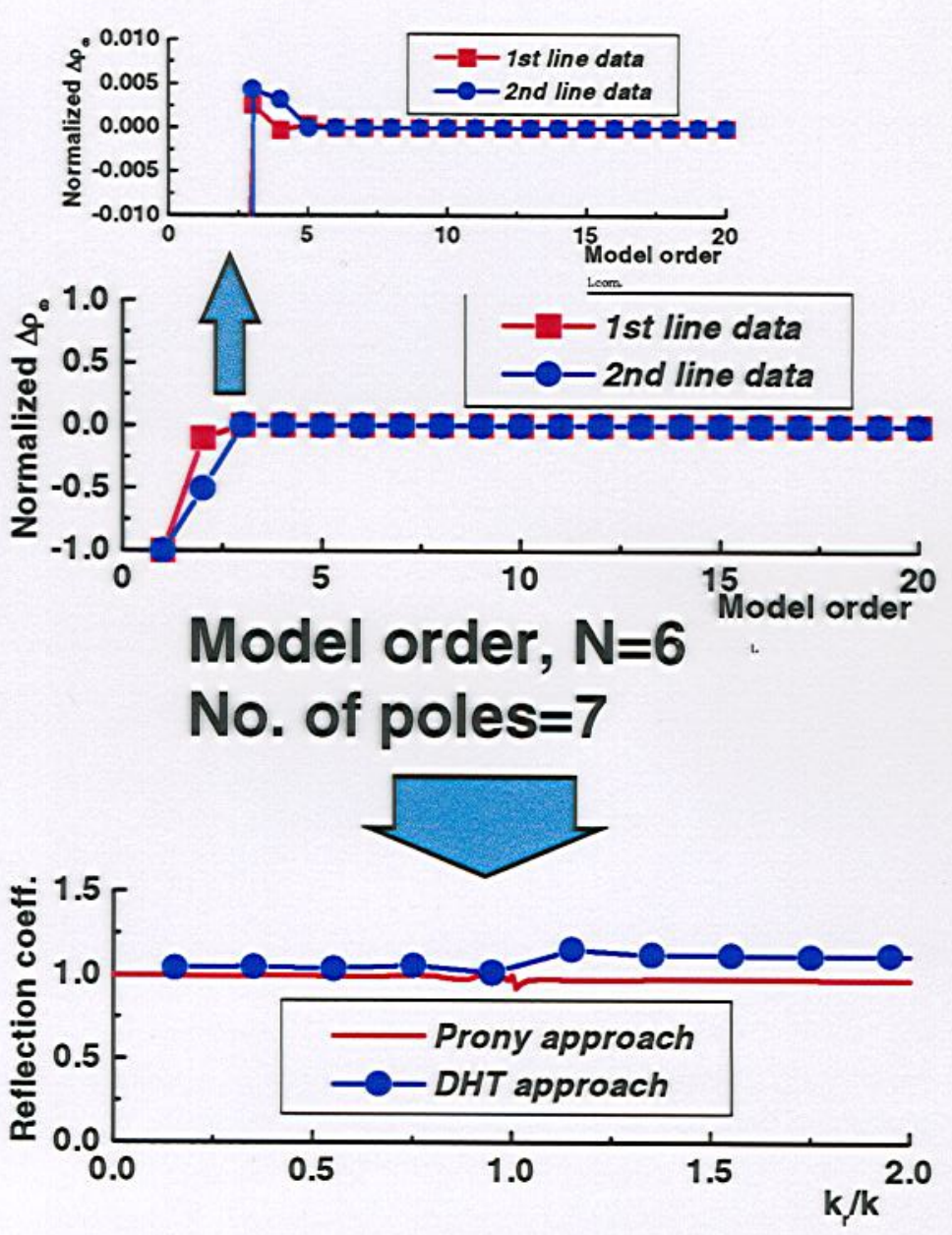

Dipole with rigid reflecting wall

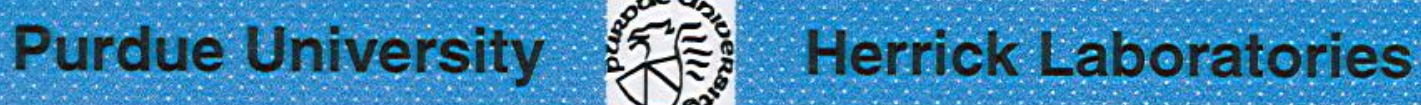




\section{EXPERIMENT}

- Experimental setup

Axisymmetric source

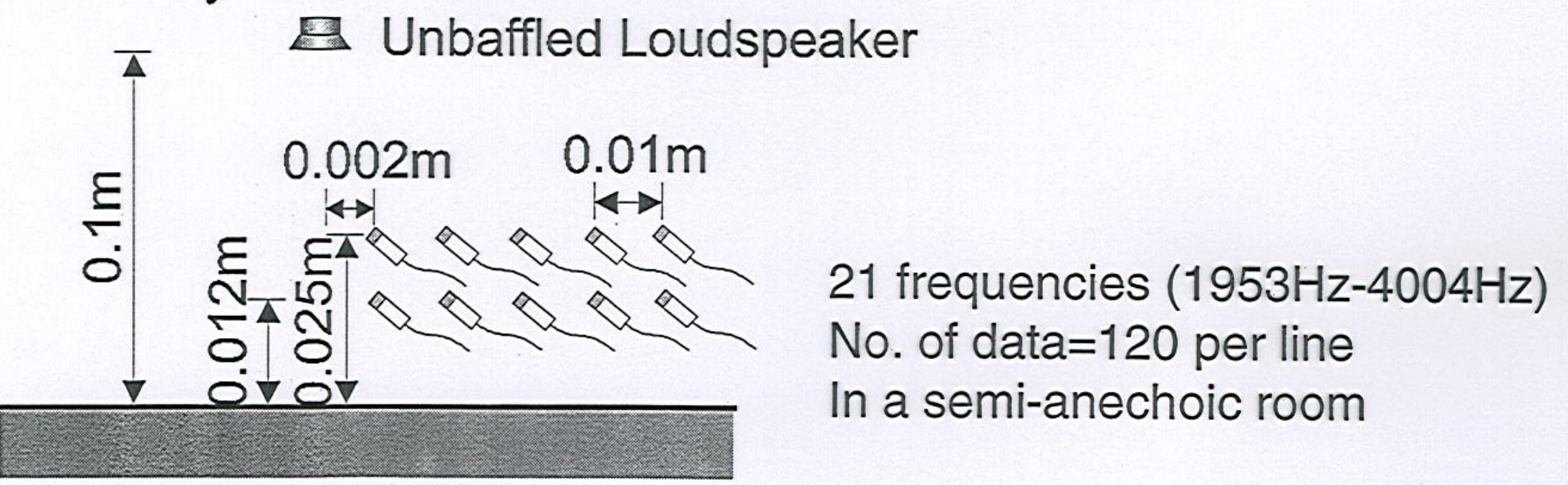

Reflecting surface, carpet

- Reflection coefficient calculation

- Discrete Hankel transform approaach

- Prony spectral estimaation approach 


\section{EXPERIMENTAL RESULTS}
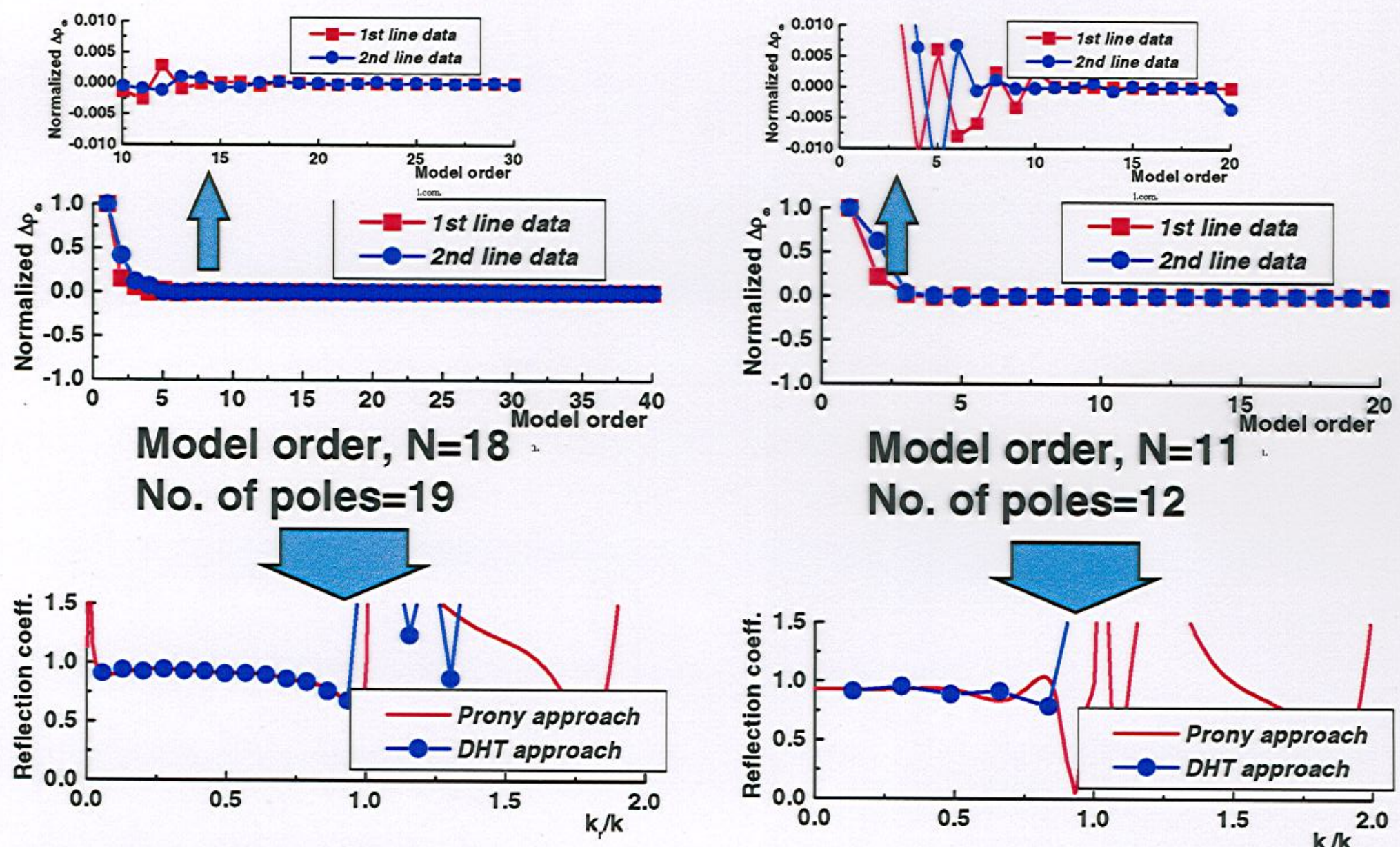

No. of poles $=12$

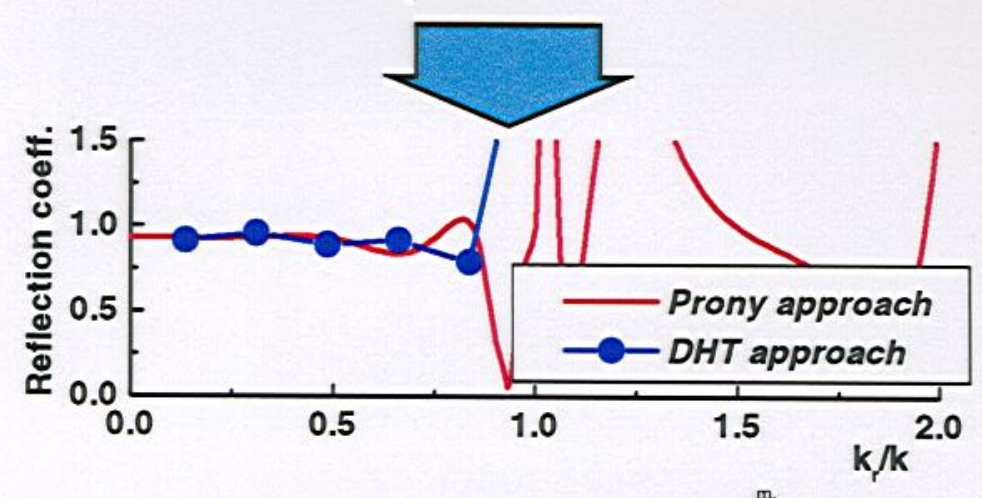

Frequency $=1953 \mathrm{~Hz}, \mathrm{~N}=120$

Frequency $=1953 \mathrm{~Hz}, \mathrm{~N}=50$ 


\section{EXPERIMENTAL RESULTS}
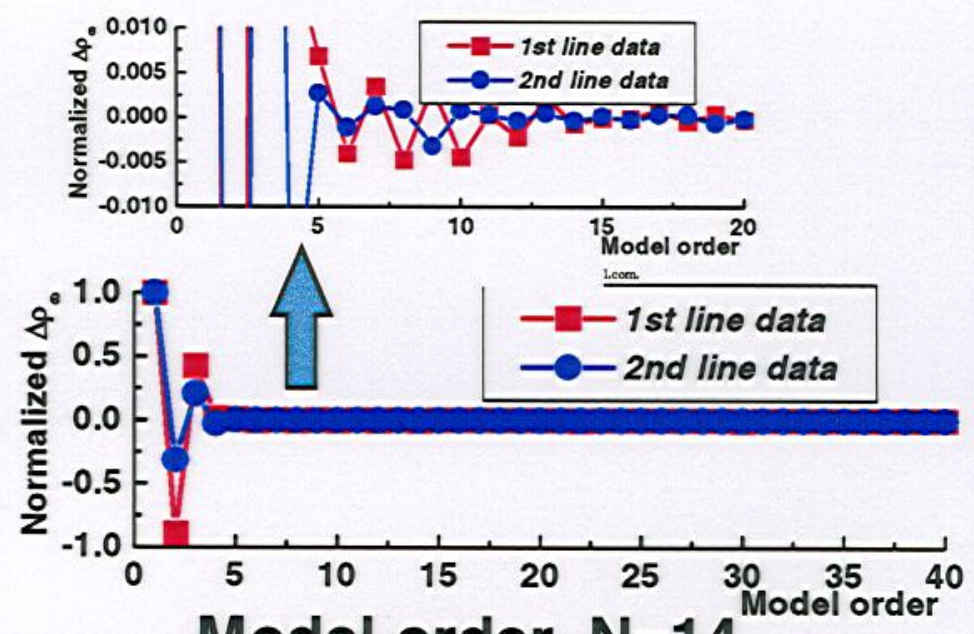

Model order, $\mathrm{N}=14$.

No. of poles $=15$

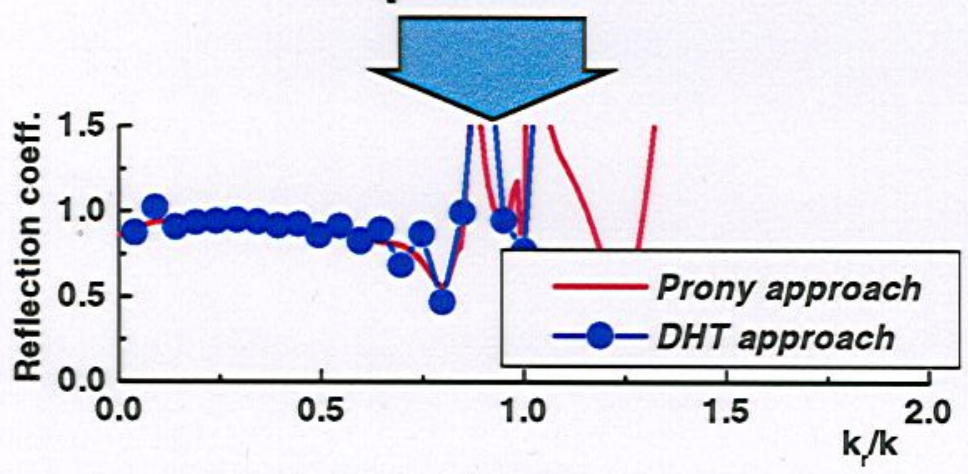

Frequency $=2832 \mathrm{~Hz}, \mathrm{~N}=120$

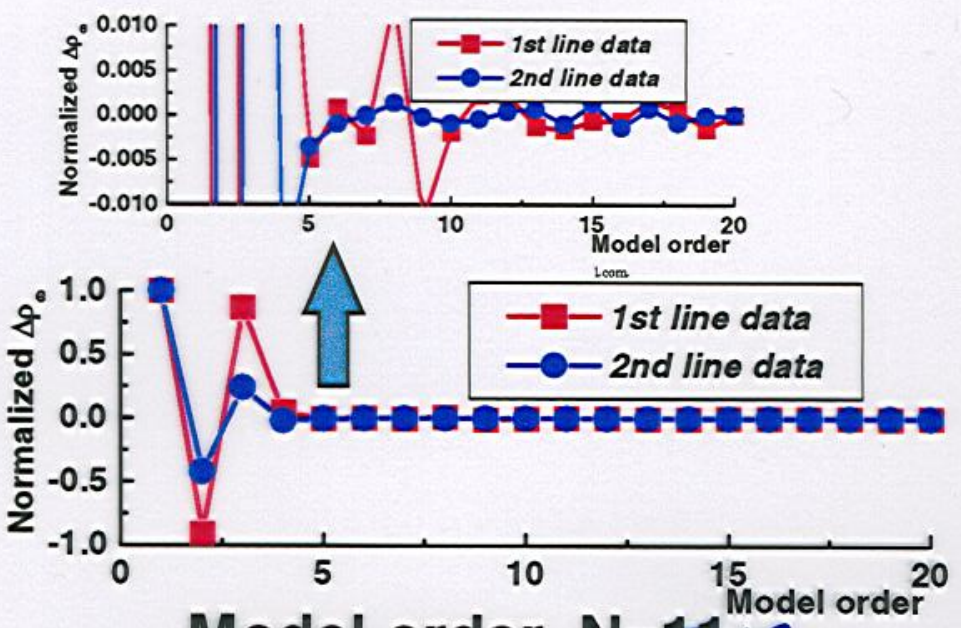

Model order, $\mathrm{N}=\$ 1 / 3$

No. of poles $=1 / 4$

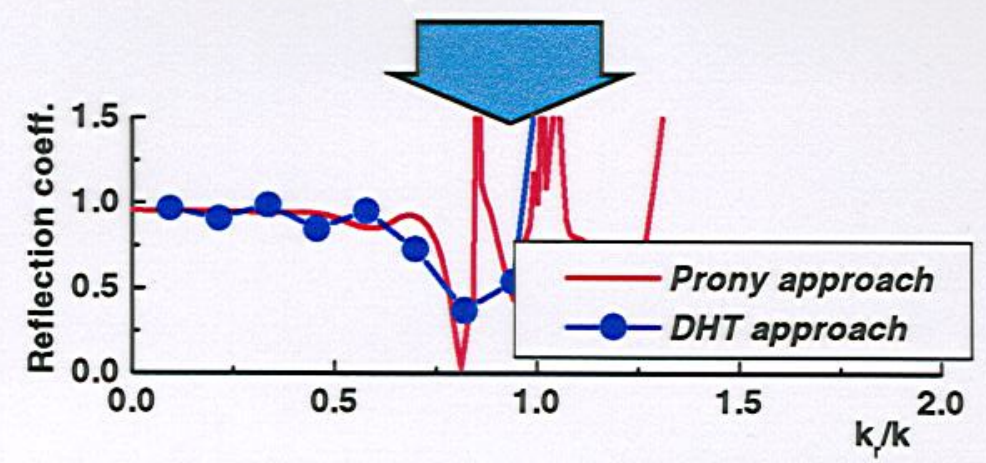

Frequency $=2832 \mathrm{~Hz}, \mathrm{~N}=50$ 


\section{CONCLUSIONS}

- Reflection coefficient measurement

- Wave decomposition theory

- 2 line microphone array

- Prony approach

- Higher spectral resolution than discrete Hankel transform approach

- Effective with less measurement points

- Less leakage due to finite aperture

- Simulation \& Experiment 


\section{APPENDIX}

- Prony model

$$
\begin{array}{rlrl}
P(r) & =\sum_{n=1}^{N} A_{n} e^{-\alpha_{n} r} \quad P(i)=\sum_{n=1}^{N} B_{n} Z_{n}^{i} & r=\delta \Delta r,(1+\delta) \Delta r,(2+\delta) \Delta r, \cdots \\
\alpha_{n}=-\frac{\log Z_{n}}{\Delta r}, A_{n}=B_{n} e^{\alpha_{n} \delta \Delta r} & \left|Z_{n}\right|<1 & \\
\tilde{P}\left(k_{r}\right) & =\int_{0}^{\infty} r P(r) J_{0}\left(k_{r} r\right) d r & \begin{array}{l}
\text { Prony method: } \\
\text { Calculate } A_{n}, \alpha_{n} \text { by use of } \\
\text { 2N or more data }
\end{array} \\
& =\sum_{n=1}^{N} A_{n} \int_{0}^{\infty} r e^{-\alpha_{n} r} J_{0}\left(k_{r} r\right) d r & \text { Reflection coefficient } \\
& =\sum_{n=1}^{N} \frac{A_{n} \alpha_{n}}{\left(\alpha_{n}^{2}+k_{r}^{2}\right)^{3 / 2}} & R\left(k_{z}\right)=\frac{-\widetilde{P}_{1} e^{-j k_{z} z_{2}}+\widetilde{P}_{2} e^{-j k_{z} z_{1}}}{\widetilde{P}_{1} e^{j k_{z} z_{2}}-\widetilde{P}_{2} e^{j k_{z} z_{1}}}
\end{array}
$$

